\title{
Human Workflow Modelling in Homogeneous Computor Networks
}

\author{
J A Johnson, A A Adekunle
}

\begin{abstract}
Maintaining consistent operational efficiency and productivity levels through effective and reliable workflow processes can be the key to businesses realising long-term profit margins. This can be difficult to achieve if the challenges that affect this dual paradigm are not fully understood. Where human input is an integral part of the business workflow, the existing methods for modelling this human interaction are too abstract in nature and are not appropriate for deriving performance characteristics about human input into the process. This paper introduces a novel approach to classifying humans for the purpose of workflow modelling. Following this classification we further demonstrate that an analytical method, such as queueing theory, can be used to extract the resource utilisation and throughput characteristics of humans engaged in a business workflow processes. The initial results from experimentations indicate that the resource utilisation and throughput levels of humans could provide insight into where performance gains and increased throughput can be made. We do not consider the unique behavioural characteristics of humans that could influence the resource utilisation or throughput rates as part of our current research.
\end{abstract}

Index Terms-Computor, Homogeneous, Human Workflow Modelling, Queueing Theory, Resource Management.

\section{INTRODUCTION}

Whilst modern business workflow processes can be predominantly automated, human workflow can form a critical part of the end-to-end business process. Businesses should seek to understand how human inter-action, efficiency and performance in workflow processes could impact on business output. A manager or key decision maker should, without prejudice, be able to establish if they are utilising their workers in the most efficient way. We found minimal evidence that the operational performance of humans, in the context of human workflow modelling, was considered.

The contribution in this paper is a novel approach to the classification of humans in the context of human workflow modelling. We use the scientifically proven formulas in queueing theory to predict the performance attributes of newly classified human entities participating in a generic workflow process.

J. A. Johnson is with the University of Greenwich, The Wolfson Centre for Bulk Solids Handling Technology, Kent ME44TB UK. (e-mail: J.A.Johnson@greenwich.ac.uk).

A. A. Adekunle is a research fellow at the University of Greenwich, Faculty of Science and Engineering (A.A.Adekunle@greenwich.ac.uk).
This paper is structured in the following manner:

Section II provides an additional glossary of terms used throughout this paper, in addition to a problem brief. Section III examines relevant literature around the problem domain. Section IV presents the proposed model. Section V discusses the methodology used and presents the modelling of workflow. Section VI analyses and discusses the experimentations and the results generated by the chosen workflow methodology. Section VII concludes.

\section{PRELIMINARY}

This section defines the additional terms used throughout this paper and presents a design brief and rationale for the work undertaken.

- Computor: - Is used in this paper to refer to a person who calculates or computes, its use differentiates a human from an artefact (i.e. computer).

- Arrival Rate: - This is the rate at which jobs or requests enter a queueing system. The lambda symbol will be used to denote the arrival rate in the queueing model used in this paper.

- Service Rate: - This is the rate at which jobs or requests can be completed. The $m u$ symbol will be used to denote the service rate in the queueing model used in this paper.

- Utilisation Rate: - In queueing theory, the utilisation rate is a measure of resource usage. The rho symbol will be used to denote the utilisation rate in the queueing model used in this paper.

Research in the area of business process modelling indicates that the modelling of human workflow can be achieved by conducting benchmark tests, using de-facto analytical models such as UML. However, an observation is that many of these analytical models that can be used for modelling human workflow fall short, in that they do not consider the performance of the human within the system. They provide a convenient way to visually represent the flow of work for humans. But these methods of workflow modelling place an emphasis on the abstract operational view. In the absence of performance based workflow analysis, the complete modelling of human workflow is missing a vital element. This paper proposes a preliminary model to quantify the performance of humans engaged in manual workflow processes. 


\section{RELATED WORK}

An extension to Service Oriented Architecture (SOA) to support human task modelling is presented in the research by [1]. The assertion is that, because many organisations continue to use their own process modelling languages and other components of software to execute the tasks required by humans, a common and unified approach to incorporating the modelling of human tasks within the SOA is needed. The view is that there is a need to accurately reproduce the tasks executed by humans to minimise errors and overall complexity.

The authors assert that there are additional human-centric details within the workflow that should be formally captured during the software development analysis phase. The authors maintain that UML has limitations in that the elements currently available cannot capture these specific details. Despite UML including UML Profiles, a lightweight extension to the modelling language where these additional details can be specified, the UML attributes Use Case and Actor still need to be extended.

The model further proposed is an extension to UML, which allows for the platform-independent modelling of human tasks with ease. A Four-step workflow process that centres on a university examination registration system is illustrated in an UML diagram. A person from the university is required to validate a registration by conducting some manual checks that the system cannot validate by itself. The Human Task Profile created from the UML profile is transformed to Business Process Execution Language (BPEL). Minimal evidence was found that the successful execution of the transformed Human Task Profile, in an appropriate environment, was achieved.

The approach to human task modelling in workflows taken by [2] was to compensate for the lack of support for human tasks in BPEL with a proposed extension to BPEL4People, using Communicating Sequential Processes (CSP) algebra. The basic model presented is a system with three processes, BPEngine, TaskEngine and People, running in parallel. Human tasks are modelled in the process People. The process TaskEngine acts as an intermediary between the business processes executed by BPEngine and the workflow processes executed by People. Because the TaskEngine process encapsulates a human task into some computerised Web service, BPEngine has no awareness of the involvement of humans as it executes its processes.

Further explanation of the interaction between these processes and the sequence of messages sent between them was expanded upon prior to the extended model being presented. This extended model, System, adds an additional process entitled TaskList. TaskList keeps track of tasks that have been claimed successfully by People. Where a task is not claimed, TaskList acts as a holding area for tasks and sits between the TaskEngine and People. The extended model also makes provision for scenarios where a task may not complete, including where a task fails and is not recoverable or requires an escalation path. A limitation to the extended model cited was a lack of progression by the system under specific or incomplete constraints, but it is maintained that such scenarios can be checked through formal verification.
The limited support for human workflow modelling afforded by BPEL cited by [3] is also touched upon in the research presented by [1]. For their research, BPEL is used to model automated workflow processes whilst a state machine is used to model the abstract human interaction in business workflow processes. It is acknowledged that other business process modelling languages cannot model human workflow to a sufficiently abstract level and this argument is used as the justification for modelling human workflow with a finite state machine.

A case study is presented where the state machine is applied to a product testing workflow. If "bugs" are identified during the product-testing workflow, the state machine allows for a human to declare a bug to be in one of the Proposed, Fixing, Rejected, Pending Info, Pending Approval or Closed states. The source language of the state machine is then transformed into the BPEL target language. Although screenshots of the framework implemented in DartFlow (a system that allows worker and organisation collaboration in the fields of research and experimentation) were illustrated, no specific details about the implementation was provided and readers were directed to the authors' pre-published papers for further information.

Bridging a gap in runtime verification of executable process models using state chart assertions is the objective of the research presented by [4]. Human input or decisionmaking in workflow processes is treated as reactive in nature. In assessing a set of desirable attributes for the formal language used to model the workflow processes, CSP notation was seen to be too mathematically complex and Petri Nets did not scale well when used to visually represent large or complex process models, due to a lack of available artifacts to represent hierarchy. In contrast to [3], the authors of [1] and [4] consider the semantics and expressive artifacts available in UML profiles to be sufficient enough "to capture human-in-the-loop decisionmaking" [4] when modelling processes. The four-step modelling process involved process selection, process analysis, process construction and construction of the state chart assertions.

To verify and validate the process model, manual testing and runtime execution monitoring were used to analyse the process flow execution during four separate test runs. Each test run was tabulated and the results compared to establish the success or failure of assertions, determine if all states were visited and to compare the execution time of each test run. It was observed that failed assertions occurred in each test run. In three of four test runs, not all states were visited. The time to execute the process increased exponentially with each test run. This could be as a result of the number of test cases being increased during each test run. This research begins to acknowledge the human in the system in an indirect manner, where the authors assert that human behaviour is a caveat to integrating the specification of human interaction into process modelling. The authors also maintain that modelling this human interaction presents challenges but, crucially, an understanding of this human interaction is required to fully verify and validate a process. The authors consider human decision-making within a process, but do not appear to consider human performance 
characteristics, and how these can be extracted, as subset of the process.

It was concluded, from the research presented, that the overall objective was to model human workflow by decomposing the tasks performed by humans and transposing these into an appropriate modelling language. Minimal evidence was found that a tactical approach to obtaining the operational performance characteristics of humans performing workflow tasks was a consideration. With no established theoretical model to capture human performance as an attribute of human workflow modelling, we present a preliminary model that we assert can be used not only to predict individual performance characteristics, but which can also be used to complement pre-existing abstract methods of human workflow modelling.

\section{PROPOSED MODEL}

An essential component of some workflow processes are the personnel tasked to complete them. These personnel could be viewed as the "computor" [10] in the context of human workflow. The concept of a computor is not too dissimilar to that of a computer. A group of human beings or a computor "network" could be solely, or collectively, responsible for a workflow process within an organisation. A network in this context refers to possible collaboration or the act of "networking" between computors to complete a task. We therefore classify a human as a computor and propose that a computor network could be modelled, to complement and improve business workflow processes by identifying areas where gains in human throughput can be achieved. The utilisation and throughput of a single, followed by multiple computors in the computor network illustrated in Figure 1, were analysed for this research. However, the approach taken could be applied to a larger computor network to measure its combined performance and throughput.

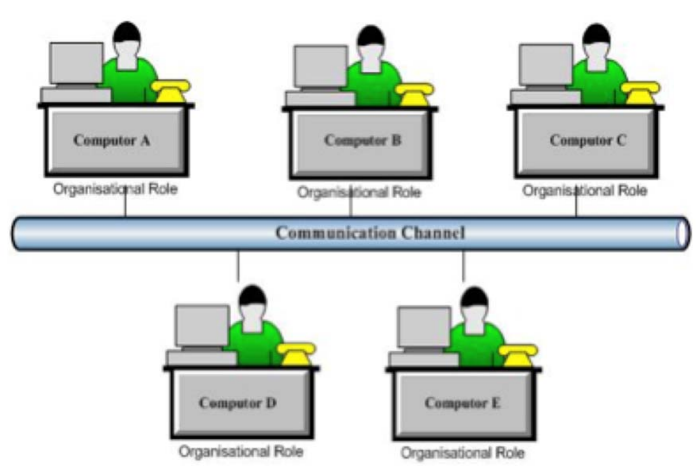

Fig 1. Computor Network for Human Workflow

All of the computors depicted in Figure 1 have an assigned role and it should be assumed that this role is a computor with the specific knowledge required to complete the workflow process. It is asserted that the way in which a computor executes processes to complete these workflow tasks bears resemblance to the way in which a computer executes processes. A computer executing processes could be illustrated as shown in Figure 2.

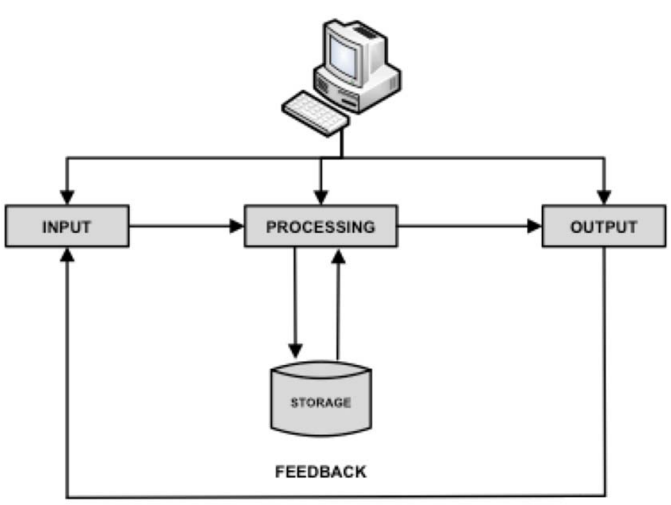

Fig 2. Computer Input-Output (I/O) Operation

To emphasise the point that a computor could operate in a similar fashion to a computer, the model illustrated in Figure 3 is proposed.

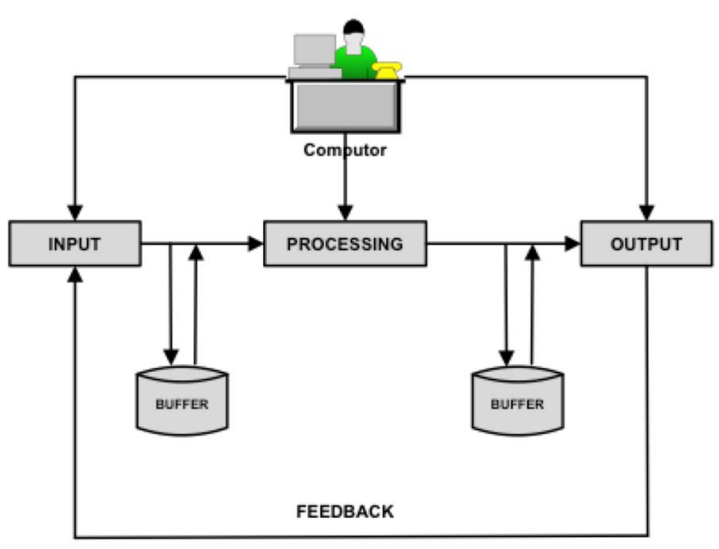

Fig 3. Computor Input-Output (I/O) Operation

For the model presented in Figure 3, an established analytical method, queueing theory, was used to analyse the performance characteristics of a single computor in isolation. This was followed by the analysis of multiple computors. Similarly to [2], the "buffer" can be viewed as a storage area to hold tasks that have yet to be executed by the computors. This further suggests that computors can be modelled, using our approach, for workflow purposes.

\section{METHODOLOGY}

The workflow process we present is service-oriented, in that customers are required to adhere to internal administrative controls of which the workflow process is a by-product. It is important to note that the computors in the network illustrated in Figure 1 are wholly reliant upon an Enterprise computer network to carry out the workflow process. For the purpose of this research it can be assumed that all computors have equal processing speed and capabilities. A computor stores the requests it receives in its buffer prior to processing. As a general rule, computors do not collaborate, but work independently of each other to complete workflow tasks. However, computors can work in parallel with their resources pooled to clear excess workflow requests where needed. We consider this novel approach to human workflow modelling to be similar to grid computing, whereby idle computors can join and leave the grid to work 
in parallel with other computors to complete workflow tasks. This grid computing configuration would differ slightly from other grid computing projects in that it would not be open and there would be a restrictions governing who could join the grid and carry out the workflow process. A workflow process was subsequently defined to provide a visual representation of the business workflow process in question. We observed that the workflow diagram provided no insight into the performance characteristics of the actors engaged in the workflow process.

A motivating scenario at this stage in our research, was to be able to establish the optimum and most efficient number of resources required to complete a given workflow process and the expected resource utilisation and throughput rates of workers. An important observation within this motivating scenario is that we consider a "computer" to be comprised of silicon and plastic and inherently devoid of any emotion. As such, emotion plays no part in a computer's ability to perform a given task. A computer's ability to complete a task would most likely be compromised by the unpredictable nature of hardware failure or software corruption. The factors impacting the ability of a "computor" to complete a task are more complex and farreaching and would require detailed analysis of basic human emotions to be of any value. We therefore do not consider these as a direct influence on the utilisation or throughput rates of computors in this scenario and consider this element to be outside of the scope of this paper. Perfect operating conditions for all computors should therefore be assumed. The only variations to the workflow process are the random nature of each request submitted. Another caveat is that the end-to-end workflow process is time-bound. All of these factors could be categorised as the latency and delay that is often experienced in traditional computer networks. An internal stakeholder triggers the workflow process by submitting internally mandated policy waiver for consideration and subsequent approval. It is the computor utilisation and throughput rates of computors participating in this workflow process that was of interest.

\section{EXPERIMENTATION AND RESULTS}

In order to establish the operational utilisation and performance the computors, both analytical and simulation methods were chosen to compare the performance characteristics of interest. Despite the Finite State Machine (FSM) being considered aptly suited to inform on human resource requirements for a given workflow, it was elected not to implement one. The justifications for this decision was that finite state machines are best suited to conditions where the outcome is fully known and transition from state to state occurs. For these tests, it was necessary to maintain a modicum of control over the results obtained to quantify the utilisation and performance of computors as accurately as possible.

\section{A. Simulated Model}

An arbitrary specified time of two hours was assigned to complete the human workflow process and this time was used as a benchmark for the analysis of the utilisation and performance of the computors. Given these factors, the workflow model was executed in simulation mode within
Visual Paradigm [3], a software design and business process modelling tool. This tool was selected as it was considered to be intuitive and easy to use. In simulation mode the time of two hours for the workflow process to execute was provided to the tool. The workflow process was executed four times and with each execution, the resources available were increased from one to a maximum of four computors executing a single workflow process. Table 1 shows the predicted resource utilisation over the duration of the workflow process when one, two, three and then four computors were assigned to a single workflow task.

TABLE 1

VISUAL PARADIGM COMPUTOR RESOURCE UTILISATION

\begin{tabular}{ccccc}
\hline \hline $\begin{array}{c}\text { Test } \\
\#\end{array}$ & $\begin{array}{c}\text { Computor 1 } \\
(\rho)\end{array}$ & $\begin{array}{c}\text { Computor 2 } \\
(\rho)\end{array}$ & $\begin{array}{c}\text { Computor 3 } \\
(\rho)\end{array}$ & $\begin{array}{c}\text { Computer 4 } \\
(\rho)\end{array}$ \\
\hline 1 & $100 \%$ & - & - & - \\
2 & $50 \%$ & $100 \%$ & - & - \\
3 & $34 \%$ & $34 \%$ & $100 \%$ & - \\
4 & $25 \%$ & $100 \%$ & - & - \\
\hline \hline
\end{tabular}

For a human workflow process, the resource utilisation of 100 per cent indicated in Table 1 would suggest that computors were busy 100 per cent of the duration of the workflow execution. This resource usage is considered to be, at best, an approximation because a computor's operating conditions may not allow for 100 per cent utilisation of the available resources for task completion. The prediction of $50 \%$ made for computor one, during the second execution, might be a better representation of the utilisation of a computor. It was also observed that during executions two, three, and four at least computor was busy 100 per cent of the duration, whilst other computors appeared to be under-utilised to varying degrees. We assert that if jobs are arriving at a particular rate and the jobs are divided equally amongst the computors, their utilisation rates should be equal. The conclusion is that the computors were not being efficiently utilised, as we could find no other plausible answer for the variation in utilisation rates during this experiment. It was clearly observed during all simulation tests that there was a period of 20 minutes when computors were idle. This 20-minute period, provided as input to the tool, accounts for the time when a Manager was performing their task, as part of the workflow process. Extended periods of idleness were observed when up to three computors were involved in the workflow execution but it could not be established, to a satisfactory degree, which computor or computors were idle, at what interval and for what length of time. It was therefore not possible to answer the questions posed as part of the motivating scenario using this method of analysis.

\section{B. Analytical Model}

To obtain any meaningful and quantifiable data, queueing theory was chosen to observe the performance of computors in our network. It is assumed that the reader is familiar with queueing theory notation and operation but the resource at [4] provides background information on the topic. Table 2 illustrates Kendall's notation [5], expressed as A/B/C/D/E, was used to define the queue type for the utilisation and performance analysis of an individual computor, or 
computors. Parameters A and B in Table 2 can follow any of the distributions illustrated in Table 3.

TABLE 2

Kendall's Notation [5]

\begin{tabular}{ll}
\hline A & Interarrival Time Distribution of Customers \\
B & Service Time Distribution \\
C & Number of Servers \\
D & Maximum Capacity of Customers in the System \\
E & Calling Population Size \\
\hline
\end{tabular}

It should be assumed that the queue service discipline is First-Come First-Served (FCFS) but other disciplines, such as Last-Come Last-Served (LCLS), Last-Come First-Serve Pre-emptive Resume (LCFS-PR) or Round Robin (RR) are possible. As this workflow process does not deal with physical customers, there was no need to consider customer behaviour. This workflow consists of "requests" and not people, although customers submit these requests. The normal rules of queueing theory do not apply for our scenario and requests cannot enter another queue if an existing queue is too long, leave the queue if service is taking too long or switch queues to obtain a quicker service; known as balking, reneging and jockeying respectively.

TABLE 3

Arrival and Service Time Distributions [7]

\begin{tabular}{cl}
\hline$M$ & Exponential (Markovian) \\
$E_{K}$ & Erlang (Parameter K) \\
$D$ & Deterministic \\
$G$ & General (Arbitrary) \\
\hline
\end{tabular}

In addition, parameters $\mathrm{D}$ and $\mathrm{E}$ in Table 3 are assumed to be infinite if they are not specified as part of the notation. Multiple $\mathrm{M} / \mathrm{M} / \mathrm{C}$ queues were created to model the policy waiver workflow process.

To compare the results to those collected during the execution of the simulated model, we recorded the values for $\rho$ or the Utilisation rate (a measure of computor resource usage) and computor throughput, which will give an indication of the output produced by a computor or computors. For these tests, it can be assumed that arrivals to the finite capacity queue, which represents a computor, are Poisson with rate $\lambda$. The service rate has a probability distribution with mean $1 / \mu$. These parameters were set to $\lambda$ $=3$ arrivals per time period and $\mu=4$ per time period. Because these queues have a finite size, any jobs that enter the queue for processing are held in the computor equivalent of storage area we refer to as the "buffer" while a computor is in service. These calculations were based on a computor, or computors, executing four workflow processes over an 8hour working day. This was reflected in the arrival rate, service rate and the maximum number of computors executing the workflow process. Figures 4 and 5 illustrate the utilisation and throughput rates at steady state produced by the queueing theory formulas, as implemented in a spreadsheet program.

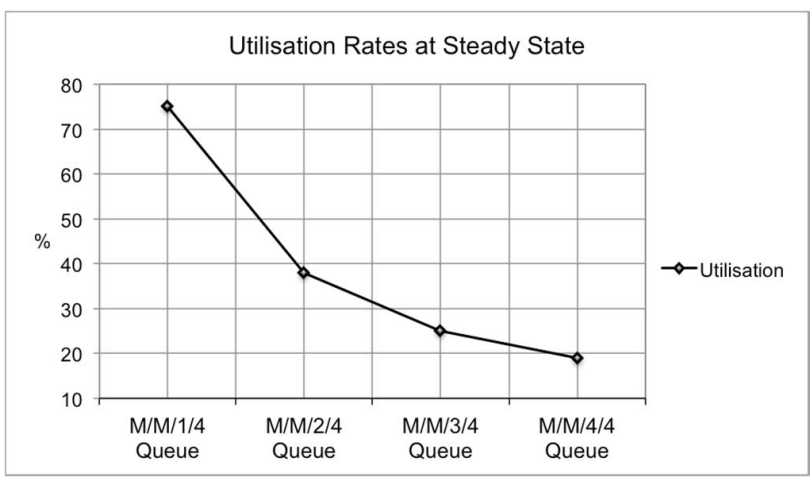

Fig 4. Computor Utilisation Rates at Steady State

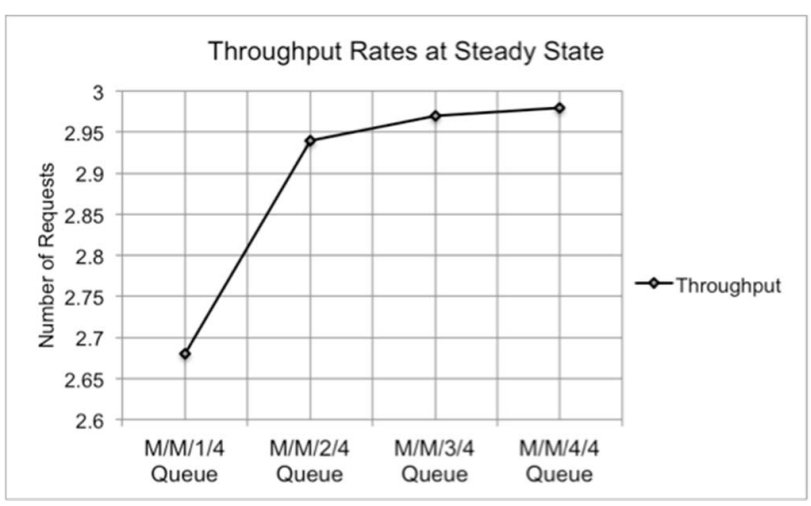

Fig 5. Computor Throughput Rates at Steady State

To verify the correctness of manually calculated queues and to compare the results shown in Figures 4 and 5 , the same parameters were defined in queues using the Operations Research Models and Methods (ORMM) Jensen Excel Add-ins [6], that includes a Queueing add-in to compute steady state performance characteristics of the Poisson queues that represent our computors. These results were identical to the queues implemented with $\mathrm{M} / \mathrm{M} / 1$ and $\mathrm{M} / \mathrm{M} / \mathrm{c}$ queueing formulas. For conciseness, Table 4 illustrates the parameters provided to the Jensen Excel Queueing Add-in for which our results were verified.

TABLE 4

JENSEN EXCEL-ADD QUEUE PERFORMANCE

\begin{tabular}{cc}
\hline Queue Station & Computor_1 \\
Arrival Rate & 3 \\
Service Rate/Channel & 4 \\
Number of Servers & 1 \\
Maximum Number in System & 4 \\
Number in Population & $* \star *$ \\
Type & $\mathrm{M} / \mathrm{M} / 1 / 4$ \\
\hline
\end{tabular}

The utilisation and throughput rates of computors were then analysed to establish if there was a range of utilisation rates that a computor could operate within to ensure that all workflow requests could be serviced. The utilisation and corresponding throughput rates were as illustrated in Figure 6 , and both sets of values were plotted together to provide an at a glance look at each parameter in parallel. 


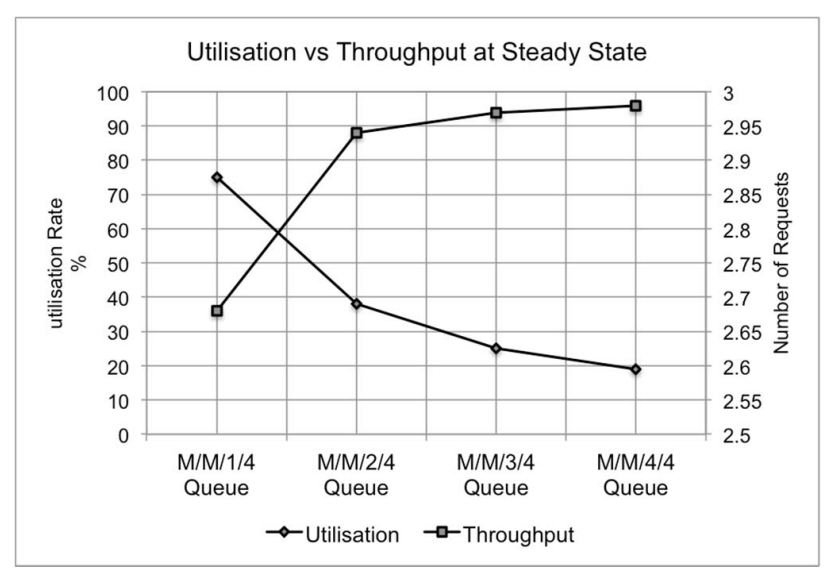

Fig 6. Utilisation vs Throughput at Steady State

The conclusion, based on the results presented in Figures 4 and 5 , is that a balance must be struck between the optimum utilisation that produces sustained throughput. In Figure 4 we observed that computor utilisation appears to experience exponential decay where one computor was busy $75 \%$ of the duration of the workflow, two computors 38, three computors $25 \%$ and finally four computors, a mere $19 \%$ of the duration of the workflow. In comparison to the simulated model, we observed equal utilisation rates for all computors where they all had the same capacity and the jobs were divided equally amongst them. In Figure 5 where throughput experiences exponential growth, there was a negligible difference in the overall throughput if two computors were working in parallel, as opposed to the maximum four available. Queueing theory also tells us that where utilisation exceeds $80 \%$, the negative effect is increased waiting time in the queue and this is applicable irrespective of whether it is a computer or human providing the service.

From the results illustrated in Figure 6 it can be observed that a relationship between the utilisation rate and throughput levels of computors during the workflow execution can be deduced. Where a single computor completes the workflow, utilisation peaked at $75 \%$ and throughput was 2.65. Where two computors completed the workflow process, utilisation dropped significantly to $38 \%$ but throughput rose to 2.95. Where three computors completed the workflow process, utilisation dropped further to $25 \%$ but throughput also increased to 2.97 . Finally, where four computors completed the workflow process, utilisation reached its lowest at $18 \%$ but throughput reached its highest at 2.98. We also observed that the total time at the station was two hours, or the time needed to complete a single workflow. All times were in minutes as for our simulated model.

It was observed that the use of a finite queue introduced increased waiting time prior to service and subsequently impacted the overall time spent in the queueing system. Figures 7 and 8 illustrate the variations in waiting time when the size of the system was set to be equal to the number of computors and when the system size was set to be greater than the number of computors available.

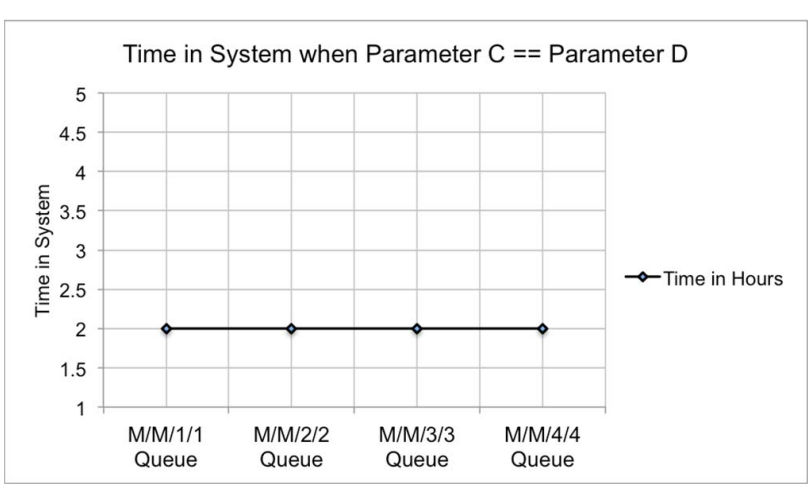

Fig 7. Time in System when $N^{o}$ of Servers $==$ System Size

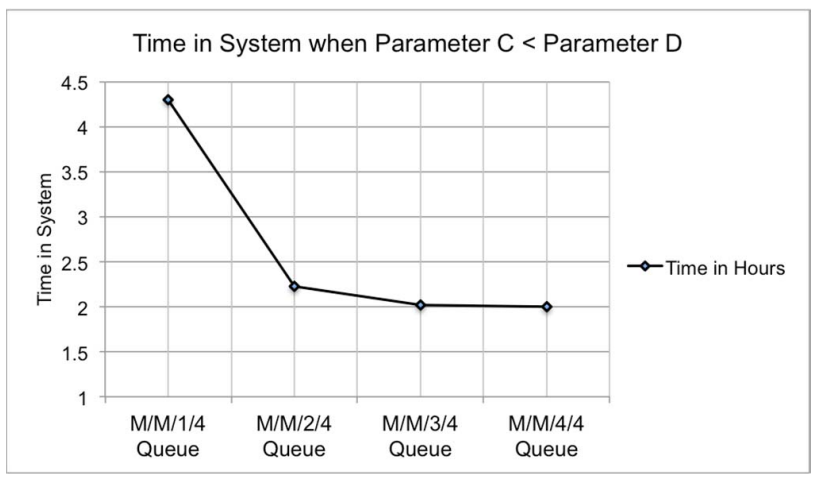

Fig 8. Time in System when $\mathrm{N}^{\circ}$ of Servers $<=$ System Size

The service times indicated in Figure 7 were expected as computors were carrying out a single workflow task in parallel and no queueing occurred with a servers == System size ratio. Figure 8 shows that the use of a buffer to hold jobs to be executed which had the impact of introducing latency into the queueing system with a No of Servers <= System Size ratio. The results illustrated in Figure 7 mirror that of infinite $\mathrm{M} / \mathrm{M} / \mathrm{C}$ queues. From an operational viewpoint and looking at these results, there could be a temptation to assign more than one computor to this workflow in the belief that increased resources will result in a quicker turnaround time, higher throughput and reduced waiting times. As Figure 8 illustrates, this theory does not necessary hold. It might be beneficial to conduct a cost/benefit analysis to determine if this is a viable option.

\section{A. Discussion}

A workflow model, implemented in a simulated environment and as an analytical model, was presented. Although some useful results were obtained, the lack of insight into how the simulation tool calculated utilisation and throughput rates was not helpful. It did not seem realistic that a single computor would be utilised at $100 \%$ for an extended period of time. The workflow model was successfully implemented with queueing theory. The results produced using this analytical method were an approximation and clearly inferences and assumptions must be made about the results obtained. Nevertheless, our approach to analysing computor utilisation and throughput can be used to create baselines for desired human performance. Some other useful computor performance characteristics were also observed, for example, the total number of jobs that were in the queue awaiting service by 
the computor or computors, along with the total time spent at the station, i.e. queueing and in service. This method of human workflow modelling is also transferrable to businesses where human operational performance is directly attributed to customer satisfaction and the business workflow process is customer-oriented in nature.

This research suggests that UML, BPEL and other highlevel languages only allow for the modelling of human workflow at an abstract level. These approaches seek to model human workflow at the strategic level and do not appear to consider the tactical approach needed to complement strategic approaches. The use of the preliminary model presented allowed human operational performance for the input into workflow processes to be modelled to some degree of accuracy. This method can be used to establish baselines for human performance as it relates to business workflow modelling.

\section{CONCLUSION}

In this positioning paper the computor, our human version of the computer, was presented. We assert that a computor has similar characteristics to that of a computer, i.e. input and output processing capabilities and a buffer or storage area. We established that computers are made of silicon and other metals and, as such, are not governed by the 'emotions' that could impact operational performance. A computer is likely to perform at a sustained rate and consistently produce the required output with a higher degree of certainty. A computor, however, can adapt to changing situations and it is this uniqueness that has warranted classifying humans as computors in the context of human workflow. We determine that due to the complex nature of human behaviour, more work is required to establish if a particular subset of human emotions do impact utilisation and throughput rates of humans engaged in manual workflow processes.

It was possible to analyse the utilisation and throughput rates of computors in a homogeneous network with our preliminary model alongside queueing theory and it was possible to extract the desired operational performance characteristics of computors engaged in the workflow process. This approach provides the tactical view of a workflow that is often absent with other workflow modelling techniques. The preliminary model presented allowed the original question posed as motivating scenario to be answered. We showed that queueing theory is one way to establish how much a worker or workers are being utilised during a given workflow process, as well as their individual or combined throughput levels. However, we accept that the model predicts the resource utilisation and throughput rates of computors only to the degree of accuracy afforded by the approach used. We also acknowledge that the preliminary model presented requires some modifications to be considered robust and as such, the results shown can only ever be approximations. The use of our preliminary model, however, does suggest that a partially accurate view of computor utilisation and throughput can be obtained, allowing resource allocation to be adjusted to meet business output production quotas.
As we have not been able to provide a complete theoretical model that takes into account human emotional state and its influences on the workflow process, we would encourage future research is directed at finding a way to formalise a strict subset of human emotions, in highly abstract terms, and incorporate these into human workflow tools or theoretical models. Our future research will be focused on expanding our model to include a small subset of human centric attributes and this is ongoing work in progress.

\section{REFERENCES}

[1] S. Link, P. Hoyer, T. Schuster and S. Abeck, "Model-Driven Development of Human Tasks for Workflows," in the Third International Conference on Software Engineering Advances, Sliema, 2008, pp. $329-335$.

[2] X. Zhao, Z. Qiu, C. Cai and H. Yang, "A Formal Model of Human Workflow," in the International Conference on Web Services, Beijing, 2008, pp. 195-202.

[3] W. Shi, J. Wu, S. Zhou, L. Zhang, Y. Yin and Z Wu, "Facilitating the Flexible Modeling of Human-driven Workflow in BPEL," in the 22nd International Conference on Advanced Information Networking and Applications - Workshops, Okinawa, 2008, pp. 1615-1624.

[4] M. Schumann, D. Drusinsky, J. B. Michael and D. Wijesekera, "Modeling Human-in-the-Loop Security Analysis and DecisionMaking Processes ," in Transactions on Software Engineering, vol. 40, 2014, pp. 154-166.

[5] (March 2016) Visual Paradigm. [Online]. Available: https://www.visual-paradigm.com/features/

[6] K. Stordahl, "The History Behind the Probability Theory and the Queuing Theory," Telenor Nordic Fixed, Oslo, 2007.

[7] A. Allen, (June, 1990), Probability, Statistics, and Queueing Theory with Computer Science Applications, Second, Ed. San Diego, California: Academic Press, Inc, [Online]. http://people.brunel.ac.uk/ mastjjb/jeb/or/queue.html

[8] P. Jensen and J. Bard, (2011, March) University of Texas. [Online]. https://www.me.utexas.edu/ jensen/ORMM/excel/

[9] G. C. Pentzaropoulos, (April, 1982), "Computer Performance Modelling: An Overview," Applied Mathematical Modelling, vol. 6, no. 2 , pp. $74-80$.

[10] $(2016$, January) Wikitionary. https:/en.wiktionary.org/wiki/computor
[Online]. 